Marek Przybyszowski', Kinga Paciorek², Weronika Zastrzeżyńska', Agnieszka Gawlewicz-Mroczka', Anna Trojan-Królikowska', Anna Orłowska ${ }^{2}$, Jerzy Soja', Wiesław Pawlik², Krzysztof Sładek ${ }^{1}$

'Il Chair of Internal Medicine, Faculty of Medicine, Department of Pulmonology, Jagiellonian University Medical College, Kraków, Poland

${ }^{2}$ Division of Radiology, Department of Allergology and Immunology, Kraków University Hospital, Kraków, Poland

\title{
Influence of omalizumab therapy on airway remodeling assessed with high-resolution computed tomography (HRCT) in severe allergic asthma patients
}

The authors declare no finacial disclosure

\section{Abstract}

Introduction: Airway remodeling is an important factor in persistent obstruction in severe asthma. High resolution computed tomography (HRCT) is an effective method of detecting changes in airway structure. Our aim was to use HRCT to assess changes in airway remodeling in patients with severe allergic asthma who are treated with omalizumab.

Material and methods: In 12 patients with severe allergic asthma, HRCT was performed before and after treatment with omalizumab. In selected bronchial airways, parameters were calculated: bronchial wall area (BA), also corrected for body surface area (BSA); percentage of wall area (WA\%); and the ratio of luminal area to total bronchial area (Ai/Ao). Clinical response to treatment was assessed using an asthma control questionnaire (ACO), asthma quality of life questionnaire (AOLO), and number of exacerbations per year. Assessment included spirometry and blood eosinophilia.

Results: Treatment resulted in significant improvement in ACO $(p=0.035)$ and AOLO $(p=0.001)$. We observed significant reduction in exacerbations per year $(p=0.002)$ and reduction of daily systemic steroid dose $(p=0.032)$. $\mathrm{FEV}_{1}$ and peripheral blood eospinophilia did not change $(p=0.846$ and $p=0.221)$. Airway dimensions ( $\mathrm{Ai} / \mathrm{Ao})$ of particular bronchi were consistent with the mean of the parameters calculated for all bronchi measured. Although we observed a significant decrease in WA $(p=$ $0.002)$ and WA/BSA $(p=0.002)$, WA $\%$ and Ai/Ao did not improve $(p=0.39$ and $p=0.49)$. We found no correlations between changes in airways and changes in spirometry or clinical parameters.

Conclusion: Despite clinical effectiveness of omalizumab, its effect on airway remodeling may be limited.

Key words: asthma, airway remodeling, HRCT, omalizumab, biological treatment

Adv Respir Med. 2018; 86: 282-290

\section{Introduction}

Severe allergic asthma is characterized by chronic inflammatory process in the airways, triggered by exposure to allergens such as dust mites, mold, and animal dander. Immunoglobulin $\mathrm{E}$ (IgE) plays a central role in asthma, not only by inducing rapid bronchoconstriction and mucus hypersecretion through type I allergic reaction, but also by migration and activation of inflammatory cells (predominantly mast cells, Th2 lymphocytes and eosinophils), leading to chronic inflammation in the airways [1]. This, in turn, leads to changes in bronchial structure, known as airway remodeling, which is characterized by reticular basement membrane (RBM) thickening, airway smooth-muscle hypertrophy, and mucus glands hyperplasia [2, 3].

Address for correspondence: Marek Przybyszowski II Chair of Internal Medicine, Faculty of Medicine, Department of Pulmonology, Jagiellonian University Medical College,

Skawińska 8, 31-066 Kraków, Poland, e-mail: mprzybyszowski@gmail.com

DOI: 10.5603/ARM.a2018.0046

Received: 17.10.2018

Copyright (C) 2018 PTChP

ISSN 2451-4934 
Omalizumab, a humanized monoclonal anti-IgE antibody, is a proven effective add-on therapy in treatment of severe allergic asthma, resulting in significant improvement in asthma control, quality of life, and reduction in exacerbations and use of systemic corticosteroids [4, 5]. Both experimental and clinical studies demonstrate the positive effect of biological treatment with anti-IgE on airway remodeling, expressed as RBM thickening or increased deposition of extracellular matrix proteins in bronchial biopsies $[3,6,7]$. However, the necessity of performing bronchoscopy with a biopsy limits the utility of this method. Consequently, chest high resolution computed tomography (HRCT) has emerged as a useful non-invasive method of airway remodeling assessment in asthma [8].

Our aim was to assess the effect of omalizumab on clinical outcomes and airway remodeling in patients with severe allergic asthma, as measured by chest HRCT in a single tertiary medical center in Poland.

\section{Material and methods}

\section{Study design}

We assessed clinical parameters and airway wall remodeling changes, measured with chest HRCT, in twelve patients with severe uncontrolled allergic asthma before and after biological treatment with omalizumab. Patients were qualified for this biological treatment program with omalizumab based on Polish National Health Fund criteria [9].

All patients were diagnosed with severe asthma according to Global Initiative for Asthma (GINA) guidelines. Allergy to perennial allergens (dust mites, cat and dog dander) was confirmed with skin prick tests or a positive serum specific $\mathrm{IgE}$ test. Serum total IgE concentration between 30 and $1500 \mathrm{IU} / \mathrm{ml}$ was required, as well as meeting at least three of the following criteria: uncontrolled asthma according to the Asthma Control Questionnaire (ACQ); Asthma Quality of Life Questionnaire (AQLQ) score < 5.0 points; three or more exacerbations in the preceding 12 months requiring starting or increasing oral corticosteroid (OCS) dose; at least one hospitalization due to asthma exacerbation in the preceding 12 months; life-threatening asthma exacerbation in the past; or persistent airway obstruction with forced expiratory volume $\left(\mathrm{FEV}_{1}\right)<60 \%$ of predicted value. Contraindications for omalizumab treatment included active smoking, pregnancy, breastfeeding, ongoing treatment with other biolo- gical or immunosuppressive agents, and presence of comorbidities worsening asthma severity.

The dose of omalizumab was calculated on the basis of serum total IgE level and the patient's weight. Omalizumab was administered subcutaneously every two or four weeks. The treatment lasted at least four months, after which patients were reevaluated. Clinical efficacy of the treatment was assessed with ACQ, AQLQ, number of exacerbations, and OCS dose. Lack of significant improvement in asthma control (defined as a drop in ACQ result by 0.5 points, drop in number of asthma exacerbations, and lowering the OCS dose at least by four milligrams of methylprednisolone equivalent), resulted in termination of treatment. Improvement of at least two of the parameters allowed the therapy to continue.

All patients had chest HRCT before and after the therapy. As the treatment period differed for each patient, changes in airway dimensions, as measured by HRCT, were correlated with treatment duration.

\section{Clinical parameters, spirometry and blood eosinophilia}

Prior to treatment, all relevant data related to asthma history were acquired, including asthma treatment, disease duration, reported allergies, and the number of severe exacerbations requiring systemic corticosteroids in the preceding 12 months. Asthma control and quality of life were evaluated with ACQ and AQLQ. The same information was collected after treatment and compared to the pre-treatment period. Allergy to perennial allergens was confirmed with allergy skin-prick tests or specific IgE testing. All patients had basic spirometry (MasterScreen, Jaeger, Wurzburg, Germany). Blood samples for peripheral blood eosinophilia and total IgE level were obtained before and after omalizumab treatment.

\section{Lung imaging}

In all study patients, thin collimation MDCT acquisition over the entire lungs at full suspended inspiration and reconstruction of contiguous $0.5 \mathrm{~mm}$ high resolution axial images was performed, both before and after treatment in the exacerbation-free period. CTs were performed with Toshiba Aquillon 64 tomograph (Toshiba Medical, Tokyo, Japan) with the following parameters: $120 \mathrm{kV}, 200 \mathrm{~mA}, 0.5 \mathrm{~mm}$ collimation. Data were reconstructed with a $0.5 \mathrm{~mm}$ interval, $512 \times 512$ matrix, and images were displayed on a window width of 1500 Hounsfield units (HU) and a window level of $-500 \mathrm{HU}$. Obtained images 
were transferred to a workstation and analyzed by a qualified radiologist who was blinded to the patient's clinical status. Using a semi-automated method and dedicated software (3D Slicer Airway Inspector, version 4.8, www.airwayinspector.acilbwh.org), a quantitative analysis of airway remodeling was performed, as described elsewhere [10].

First, we identified bronchi, which promised the most accurate measurement of airway diameters. Segmental, subsegmental and sub-subsegmental $\left(3^{\text {rd }}, 4^{\text {th }}\right.$ and $5^{\text {th }}$ generation $)$ bronchi to first and tenth segments of the right and left lung were chosen for further measurements. On cross-sectional scans, the place of measurement was selected by the radiologist, then software automatically calculated airway diameters. The following parameters were measured in each bronchus: wall area (WA); WA corrected for body surface area (WA/BSA); and the luminal area to outer airway area ratio (Ai/Ao). Finally, in each patient, 8 to 15 bronchi were analyzed. We selected one bronchus from each investigated generation, which was reliably visualised in each patient: the tenth left basal segment (L10); first right apical subsegment (R1.a); and the first right apical sub-subsegment (R1.a.1). In addition, the mean value of Ai/Ao in all measured bronchi and the sum of WA and WA/BSA of the three selected bronchi were calculated.

Inter-observer reproducibility was assessed by having another radiologist independently analyze the same parametrs in R1.a in five randomly selected patients. The mean results were compared using t-test.

\section{Statistical analysis}

All data were analyzed using Statistica software (ver. 12, StatSoft). Values were expressed as means with standard deviations (SD - in parentheses). For comparisons of changes in clinical parameters, pulmonary function tests, inter-observer mesurement reproducibility and airway diameters pre- and post- treatment, the t-test for related samples was used. Correlations between changes in airway diameters, clinical parameters and lung function for normal distributions were made using the Pearson's linear correlation coefficient. The significance of the differences between alternative methods of airway remodeling assessment (measurement of Ai/Ao in single bronchus, three selected bronchi or the mean of all measured bronchi as well as pre- and post-treatment changes of those parameters) was verified by univariate analysis of variance (ANOVA). In case of significant difference, a post-hoc
Scheffe test was used successively to identify specific comparison pairs differing from each other. A $p$ value $=0.05$ was considered significant.

\section{Results}

Characteristics of study subjects are presented in Table 1. All patients met the inclusion criteria of Polish National Health Fund program. None of the patients actively smoked. After four months of biological treatment, one patient was disqualified from further therapy due to poor response. Eleven patients were evaluated as good responders and continued the treatment for different periods of time. The mean treatment period in the studied group was 30.8 months ( \pm 16.1 ), and the mean dose of omalizumab was $475 \mathrm{mg}$ $( \pm 277.7)$ for four weeks.

\section{Clinical parameters}

We observed significant improvement in asthma control after treatment with omalizumab. The mean ACQ result dropped from 3.14 to 2.4 points $(p=0.035)$. At the same time, significant improvement in quality of life was observed, with the mean AQLQ score rising from 3.54 to 4.68 points $(\mathrm{p}=0.001)$. Mean exacerbation rate declined significantly from 5.5 to 1.5 per year $(p=0.002)$. During therapy, the mean daily OCS dose was significantly reduced from 14.67

\section{Table 1. Characteristics of study subjects}

\begin{tabular}{|c|c|}
\hline Parameter & All $(n=12)$ \\
\hline Female, $\mathrm{n}(\%)$ & $10(83.33)$ \\
\hline Age (yrs), mean (SD) & $47.9(8.58)$ \\
\hline BMI $\left(\mathrm{kg} / \mathrm{m}^{2}\right)$, mean (SD) & $25.52(4.84)$ \\
\hline $\mathrm{BSA}\left(\mathrm{m}^{2}\right)$, mean $(\mathrm{SD})$ & $1.83(0.26)$ \\
\hline Asthma duration (yrs), mean (SD) & $29.42(16.0)$ \\
\hline Treatment duration (months), mean (SD) & $30.75(16.09)$ \\
\hline $\mathrm{FEV}_{1} \%$ predicted, mean (SD) & $73.07(18.43)$ \\
\hline $\mathrm{FEV}_{1} \% / \mathrm{FVC}$ after bronchodilator, mean (SD) & $63.77(9.88)$ \\
\hline Fixed obstruction, n (\%) & $10(83.33)$ \\
\hline OCS users, $\mathrm{n}(\%)$ & $12(100)$ \\
\hline OCS (mg/day), mean (SD) & $14.67(8.23)$ \\
\hline ICS high dose ${ }^{a}, \mathrm{n}(\%)$ & $12(100)$ \\
\hline Omalizumab dose (mg/4 weeks), mean (SD) & $425(277.57)$ \\
\hline $\operatorname{lgE}(\mathrm{IU} / \mathrm{uL})$, mean (SD) & $228.69(134.08)$ \\
\hline $\begin{array}{l}\text { Peripheral blood eosinophilia (cells/uL), } \\
\text { mean (SD) }\end{array}$ & $202.5(127.36)$ \\
\hline
\end{tabular}


Table 2. Comparison of clinical parameters, spirometry and blood eosinophilia before and after treatment with omalizumab

\begin{tabular}{lccc}
\hline Parameter & Before treatment & After treatment & p \\
\hline ACQ, mean (SD) & $3.14(0.46)$ & $2.4(1.06)$ & 0.035 \\
AQLO, mean (SD) & $3.54(0.77)$ & $4.68(0.65)$ & 0.001 \\
Exacerbations per 12 months, mean (SD) & $5.5(2.94)$ & $1.5(1.78)$ & 0.002 \\
FEV ${ }_{1}$ predicted, mean (SD) & $73.07(18.43)$ & $72.18(19.22)$ & 0.846 \\
FEV ${ }_{1}$ /FVC after bronchodilator, mean (SD) & $63.77(9.88)$ & $67.19(8.62)$ & 0.160 \\
OCS (mg/day), mean (SD) & $14.67(8.23)$ & $10.50(11.06)$ & 0.032 \\
Peripheral blood eosinophilia (cells/uL), mean (SD) & $202.5(127.36)$ & $261.67(182.60)$ & 0.221 \\
\hline
\end{tabular}

milligrams of methylprednisolone equivalent to 10.5 milligrams $(\mathrm{p}=0.032)$, while the mean dose of inhaled corticosteroids (ICS) did not change significantly.

\section{Spirometry}

Mean $\mathrm{FEV}_{1}$ did not change significantly after treatment $(\mathrm{p}=0.848)$. Mean $\mathrm{FEV}_{1} / \mathrm{FVC}$ improved insignificantly from $63.77 \%$ to $67.19 \%$ ( $p=$ 0.160). However, in three patients, we observed an increase in the $\mathrm{FEV}_{1} / \mathrm{FVC}$ ratio, exceeding $70 \%$, as compared to the pre-treatment period.

\section{Blood eosinophilia}

We observed an insignificant increase in peripheral blood eosinophilia from 202.5/ul to $261.57 / \mathrm{ul}$ following treatment $(\mathrm{p}=0.221)$. Comparisons between pre- and post-treatment clinical parameters, lung function tests and eosinophilia are presented in Table 2 .

\section{Airway remodeling assessed with HRCT}

The total WA and WA/BSA of three selected bronchi were significantly reduced in comparison to the pre-treatment period: $124.53 \mathrm{~mm}^{2}$ versus $105.40 \mathrm{~mm}^{2}$, $[\mathrm{p}=0.002]$, and $68.26 \mathrm{~mm}^{2} / \mathrm{m}^{2}$ versus $58.16 \mathrm{~mm}^{2} / \mathrm{m}^{2}$, [p $\left.=0.002\right]$ respectively. No significant changes were observed in Ai/Ao of three selected bronchi, and in $\mathrm{Ai} / \mathrm{Ao}$ of all bronchi measured ( 0.36 versus 0.35 [ $p=0.565]$, and 0.35 versus 0.34 [ $p=0.491]$, respectively) (Fig. 1). Changes in WA and WA/BSA of selected bronchi did not correlate with changes in $\mathrm{FEV}_{1}$, ACQ, AQLQ, exacerbation rate, treatment time or asthma duration (Table 3). Selected correlations are shown in Figure 2.

In the univariate ANOVA and the post-hoc Scheffe test, mean Ai/Ao of all measured bronchi was not statistically different from Ai/Ao of individual bronchi (Table 4). No significant differenc- es were observed between pre- and post-treatment changes of $\mathrm{Ai} / \mathrm{Ao}$ of individual bronchi, mean Ai/Ao of three selected bronchi, or all bronchi measured $(\mathrm{p}=0.774)$.

Inter-observer reproducibility was satisfactory. No significant differences were observed in mean WA (34.11 $\mathrm{mm}^{2}$ vs $31.98 \mathrm{~mm}^{2}[\mathrm{p}=0.428]$ ), WA/BSA $\left(20.29 \mathrm{~mm}^{2} / \mathrm{m}^{2}\right.$ vs $19.22 \mathrm{~mm}^{2} / \mathrm{m}^{2}$ [p = $0.481]$, WA\% (62.65\% vs $61.68 \%[p=0.308])$ and $\mathrm{Ai} / \mathrm{Ao}(0.393$ vs $0.383[\mathrm{p}=0.522])$ of R1a measured by two independent radiologists.

\section{Discussion}

Results of our study show, that in real-life conditions, omalizumab is an effective add-on therapy for patients with severe allergic asthma, which remains uncontrolled despite intensive treatment. We confirmed that biological treatment with omalizumab significantly improves asthma control, asthma-related quality of life and lowers exacerbation rate. In addition, the treatment allowed for significant reduction in daily OCS dose in the studied group. Our findings align with results of previous studies on this topic [5, 11].

However, despite significant improvement in clinical outcomes, treatment with omalizumab only partially influenced airway wall remodeling. Decrease in WA and WA/BSA, while significant, was not associated with improvement in the airway lumen area to bronchial wall area ratio (Ai/Ao). FEV ${ }_{1}$ and peripheral blood eosinophilia remained unchanged. Moreover, we did not observe significant correlations between changes in bronchial wall area and $\mathrm{FEV}_{1}$.

Our study is one of the few published studies assessing the effect of omalizumab therapy on airway remodeling assessed with chest HRCT. Hoshino et al. demonstrated a spectacular decrease in bronchial wall area with increase in luminal area during omalizumab treatment, 


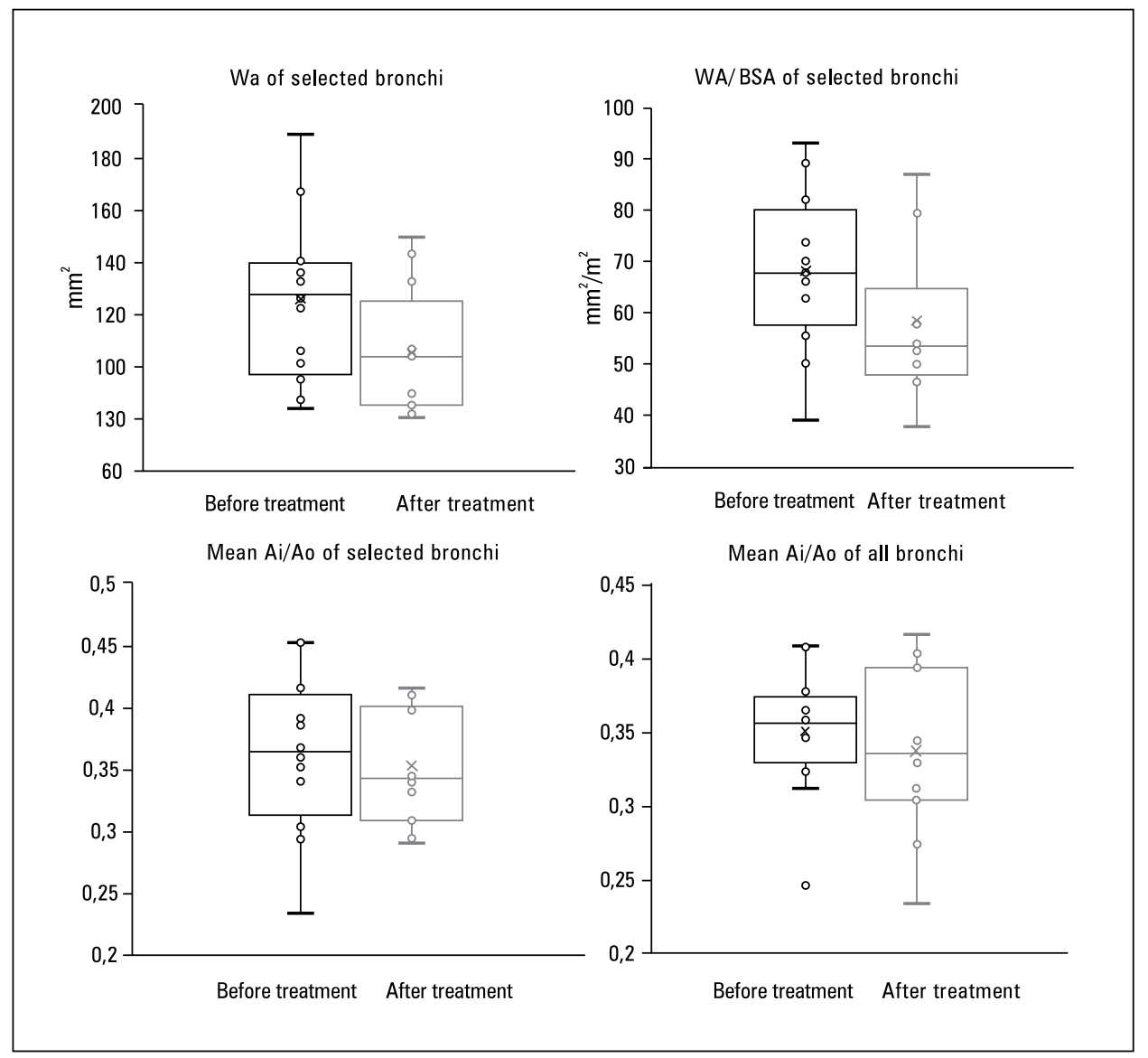

Figure 1. Changes in bronchial wall area after biolgical treatment with omalizumab

Table 3. Correlations between changes in airway dimensions and clinical parameters

\begin{tabular}{|c|c|c|c|c|c|c|c|}
\hline Parameter & $\Delta \mathrm{FEV}_{1}$ & $\begin{array}{l}\Delta \mathrm{FEV}_{1} / \\
\mathrm{FVC}\end{array}$ & $\triangle \mathrm{ACO}$ & $\triangle$ AOLO & $\begin{array}{l}\Delta \text { number of exacer- } \\
\text { bations } / 12 \text { months }\end{array}$ & asthma duration & treatment duration \\
\hline \multicolumn{8}{|c|}{$\Delta$ WA of selected bronchi } \\
\hline $\mathrm{R}$ & 0.127 & 0.501 & -0.006 & -0.363 & 0.566 & 0.087 & -0.302 \\
\hline $\mathrm{p}$-value & 0.695 & 0.097 & 0.986 & 0.247 & 0.055 & 0.788 & 0.340 \\
\hline \multicolumn{8}{|c|}{$\Delta \mathrm{WA} / \mathrm{BSA}$ of selected bronchi } \\
\hline $\mathrm{R}$ & 0.092 & 0.469 & -0.013 & -0.380 & 0.555 & 0.101 & -0.193 \\
\hline $\mathrm{p}$-value & 0.775 & 0.124 & 0.969 & 0.223 & 0.061 & 0.756 & 0.547 \\
\hline
\end{tabular}

while airway remodeling parameters in patients with asthma treated with ICS only remained unchanged. He also observed a significant increase in $\mathrm{FEV}_{1}$ and reduction of eosinophil count in induced sputum among biologically treated patients [12]. Similarly, Tajiri et al. [13] observed improvement in WA\%, which also correlated with decrease in sputum eosinophils and the level of exhaled nitric oxide.

Findings in these studies are not consistent with our results, likely due to several noteworthy differences between them and our study. First, mean duration of asthma (30 years) was almost twice as long in our study as compared to Hoshino's work (16.3 years) and Tajiri's (16.2 years). As proven in previous studies, airway remodeling severity is proportional to disease duration $[8,14,15]$. Observations by Boulet et al. [16] indicate that remodeling occurs at early stages of asthma, even with short history of symptomatic disease. Additionally, changes in airway structure are not fully reversible with ICS treatment. 


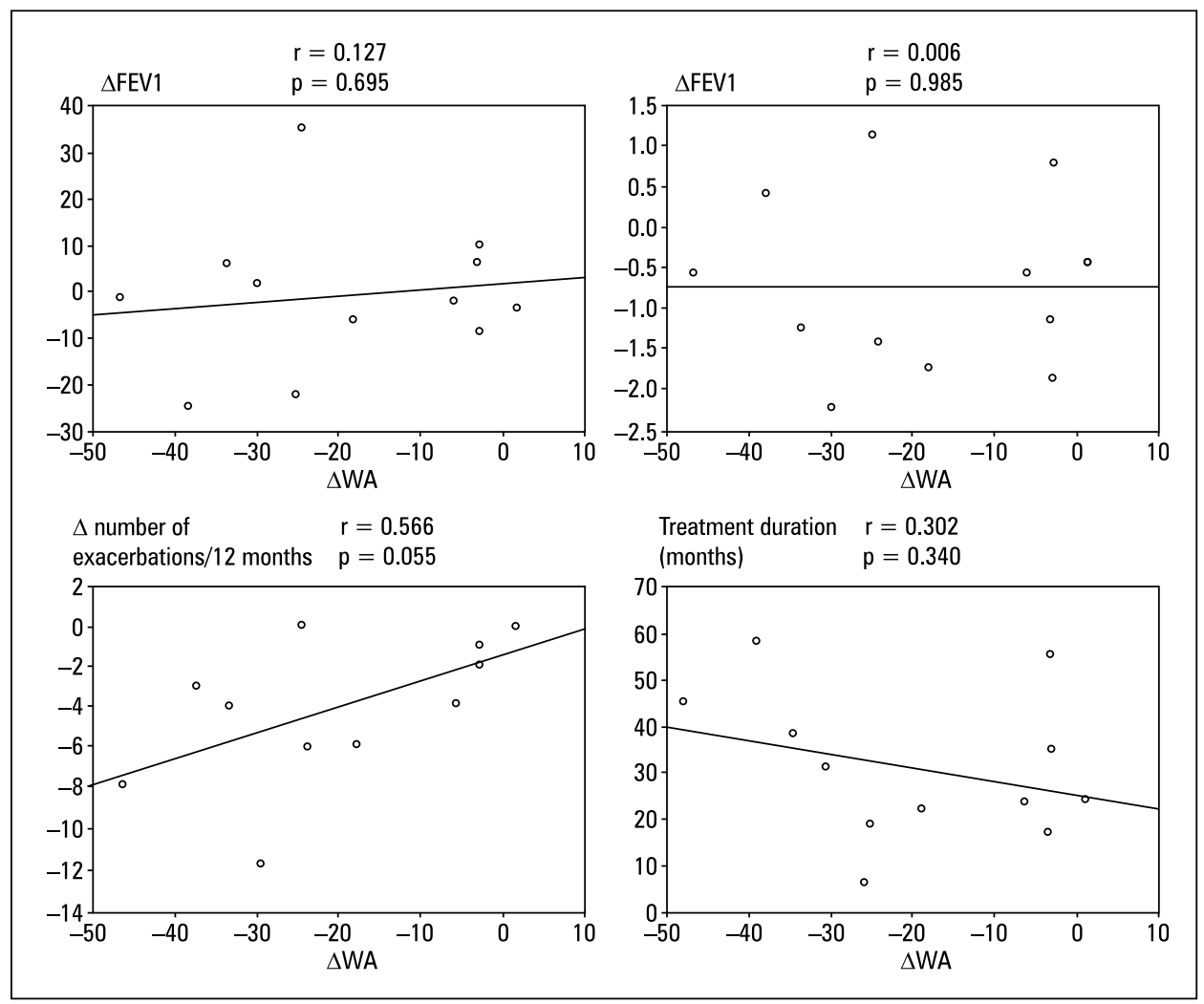

Figure 2. Selected correlations between changes in airway dimensions and clinical parameters

Table 4. Comparison of pre-treatment airway remodeling parameters with checking for significant differences between them

\begin{tabular}{lccccc}
\hline $\begin{array}{l}\text { Post-hocTest Scheffe } \\
\text { (p-value) }\end{array}$ & $\begin{array}{c}\text { Mean Ai/Ao of } \\
\text { three selected } \\
\text { bronchi }\end{array}$ & $\begin{array}{c}\text { Mean Ai/Ao of } \\
\text { all bronchi }\end{array}$ & Ao/Ai L10 & Ao/Ai R1a & Ao/Ai R1.a.1 \\
\hline Mean Ai/Ao of three selected bronchi & - & 0.995 & 0.225 & 0.960 & 0.048 \\
Mean Ai/Ao of all bronchi & 0.995 & - & 0.100 & 0.824 & 0.122 \\
Ao/Ai L10 & 0.225 & 0.100 & - & 0.616 & 0.000 \\
Ao/Ai R1a & 0.960 & 0.824 & 0.616 & - & 0.007 \\
Ao/Ai R1.a.1 & 0.048 & 0.122 & 0.000 & 0.007 & - \\
\hline
\end{tabular}

Therefore, the effect of anti-inflammatory treatment on airways profoundly remodeled by a long-term inflammatory process is likely to be limited. Second, 10 of the 12 studied patients had persistent obstruction, confirmed with a $\mathrm{FEV}_{1} /$ /FVC ratio below $70 \%$ after use of a bronchodilator. As previously shown, the degree of airway obstruction is linked with increase in airway thickness, especially in distal bronchi $[8,17,18]$. Significant airway thickening was observed in both proximal and distal bronchi among patients with asthma who have fixed obstruction, despite anti-inflammatory treatment with inhaled or sys- temic steroids [19]. Although some studies show positive effects of anti-inflammatory treatment with ICS or ICS + long acting beta agonists on airway remodeling parameters in chest HRCT $[20,21]$, they were performed on patients with mild to moderate asthma who were steroid-naïve. Finally, our studied group consisted exclusively of patients with long-term severe asthma with persistent obstruction and uncontrolled disease despite long-term treatment with high doses of ICS or OCS. As proven, airway remodeling advancement is closely linked to disease severity [22]. Therefore, we conclude that those factors may be 


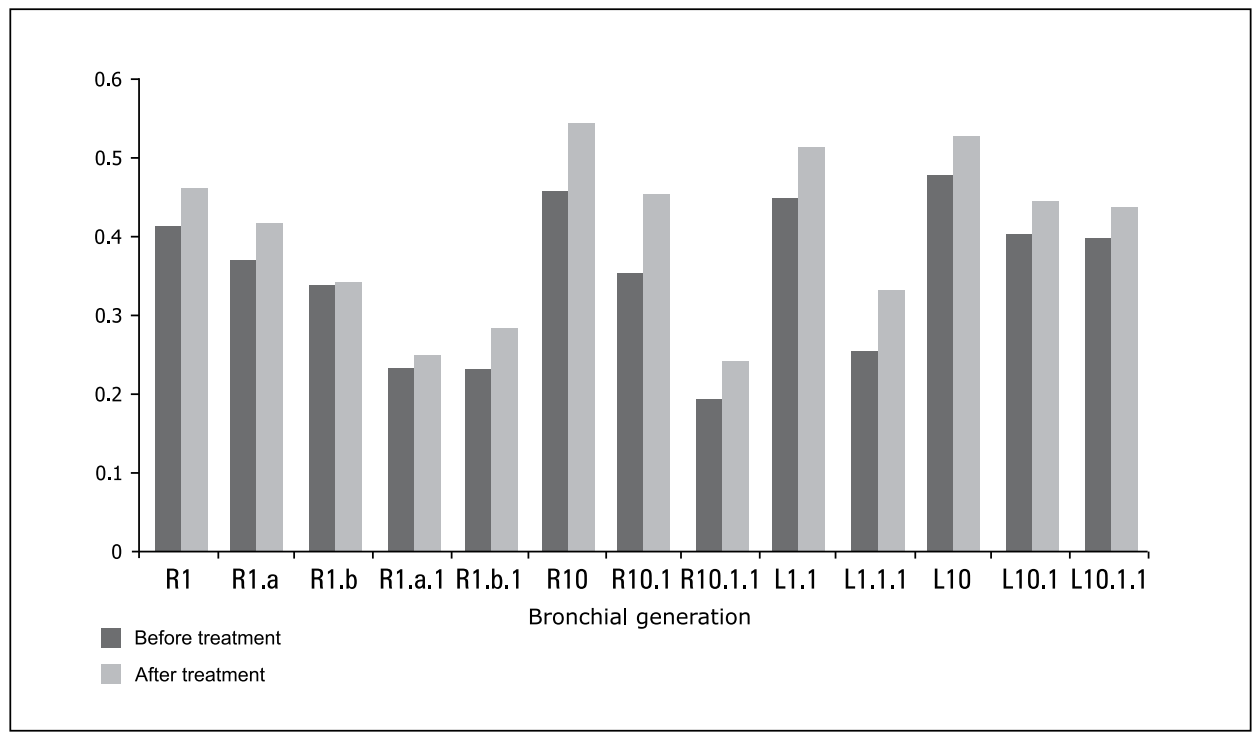

Figure 3. Changes of Ai/Ao in selected patient's bronchi measured with chest HRCT before and after treatment with omalizumab

responsible for only partial response to treatment in terms of changes in airway remodeling.

In many studies, different parameters of airway remodeling measured in chest HRCT were analyzed. Some authors indicate absolute parameters such as WA as the most accurate, since relative parameters (e.g. WA\%, Ai/Ao) may be dependent on lung volume, bronchial dilation and presence of bronchiectases [8, 23]. However, thickness of the bronchi is affected by bronchial size and does not inform about the inside of the bronchus. A more precise determination of airway condition might come from evaluating relative parameters - bronchial thickness and lumen diameter - as they reflect airway narrowing. The studies also vary in selection of bronchi for airway measurements. In our study, we measured between 8 to 15 bronchi in each patient. In all of them, we observed very consistent changes in remodeling parameters (Fig. 3). The mean Ai/Ao of all bronchi was not significantly different from $\mathrm{Ai} /$ Ao of particular bronchi, therefore, this could be reflective of airway dimensions in a patient. In our opinion, lack of improvement in the ratio of lumen area to bronchial area (Ai/Ao) corresponds with unchanged $\mathrm{FEV}_{1}$ and features of fixed obstruction within the studied group.

In four patients, we observed marked improvement of all remodeling parameters. The pre- to post-treatment changes in HRCT scans of patients with improvement of airway diameters, as well as of patients without improvement, are shown on Figure 4. In those patients, however, lung function, expressed as $\mathrm{FEV}_{1}$, did not improve. It has to be taken into consideration that the influence of omalizumab on small airways in our patients is unknown. Previous research by Little et al. [24] demonstrated that the degree of remodeling corresponded with $\mathrm{FEF}_{25-75}$ rather than with $\mathrm{FEV}_{1}$. This may reflect significant impact of the small airways remodeling on lung function. Similar results were provided by Hoshino, who observed stronger correlations between lung function and remodeling in distal rather than in proximal airways [17]. Unfortunately, because of our small patient sample, we were unable to investigate significant differences between patients who improved in HRCT and those who did not respond well to therapy.

We observed no significant change in peripheral blood eosinophilia during treatment. Several studies analyzed the effect of IgE on eosinophils. As shown previously, IgE contributes to airway inflammation not only through mast cell degranulation and direct influence on the airways, but also through eosinophil activation $[25,26]$. Anti-IgE treatment was shown to be particularly effective in patients with asthma with high blood eosinophil count [27]. Djukanovic et al. [28] found that omalizumab decreased eosinophilic infiltrates in bronchial mucosa. On the other hand, in the recent STELLAIR study, efficacy of anti-IgE therapy was similar in patients with high and low blood eosinophilia, and initial eosinophil count was not predictive of treatment outcome [29]. Discrepancies between studies suggest that the relationship between IgE, eosinophilic inflammation and airway remodeling requires further investigation. 


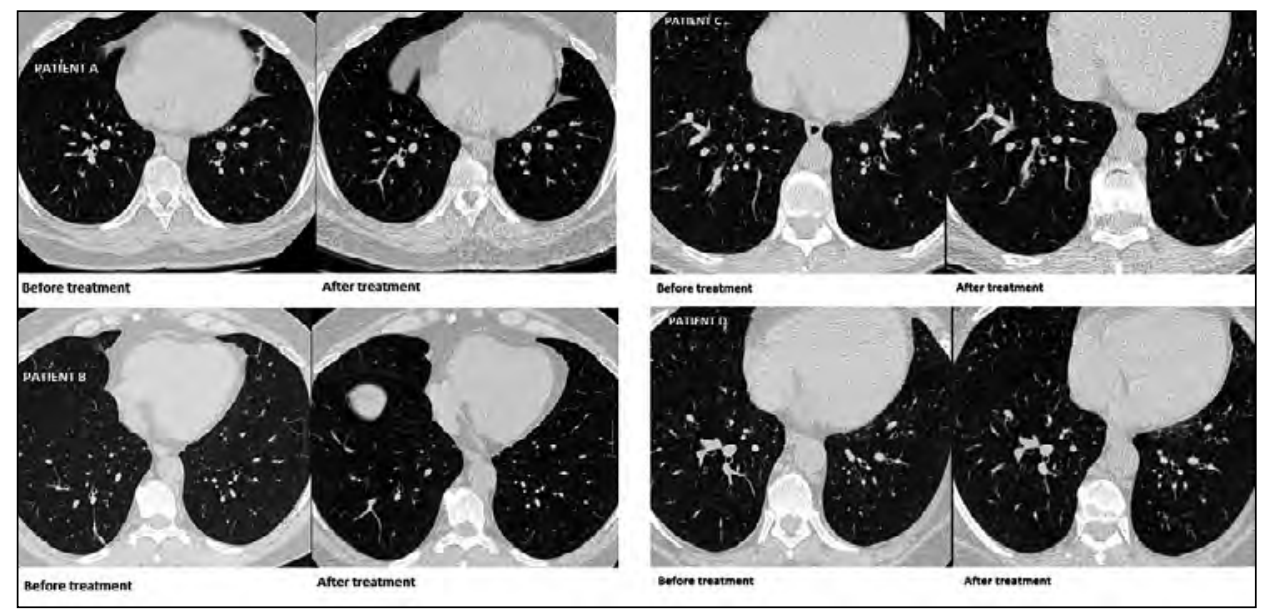

Figure 4. Chest $C T$ scans of two patients with marked improvement in airway remodelling ( $A$ and $B$ ) and two patients without improvement (C and $D)$ — comparison before and after the treatment

Our study has some limitations. First is the small number of patients with asthma, which did not allow us to investigate subgroups of patients with various airway remodeling changes. This may have biased our statistical analysis. Second, biological treatment duration varied significantly between our patients, due to individual patient's decisions and disqualification of one patient because of lack of response to therapy. However, our results indicate that treatment duration did not correlate with observed bronchial wall area changes. It may reflect the fact that remodeling in patients with long term severe asthma is an advanced process, [15] which cannot be fully reversed with either short or long term biological treatment. Such conclusion is also supported by the fact that we did not observe improvement in spirometry. Third, our group consisted exclusively of patients with severe uncontrolled asthma with long-standing disease, frequent exacerbations, and high ICS and OCS intake. These were the only patients who could qualify for omalizumab treatment in accordance with the requirements of the National Health Fund. However, patients with severe allergic asthma are a heterogeneous group [30], and therefore, large studies on severity-stratified patients with asthma are needed. Despite those limitations, we have thoroughly evaluated the influence of omalizumab treatment on clinical parameters and airway remodeling among patients with long-term severe allergic asthma in a single tertiary medical center.

\section{Conclusions}

The addition of omalizumab to standard asthma treatment improved asthma control, asthma-related quality of life, OCS intake, and exacerbation frequency in patients with long-term severe allergic asthma. However, the influence of biological treatment on airway remodeling was only partial and without effect on spirometry and blood eosinophilia. The influence of biological treatment on airway remodeling in different groups of patients with asthma needs further studies.

\section{Conflict of interest}

The authors declare no conflict of interest.

\section{References:}

1. Owen CE. Immunoglobulin E: role in asthma and allergic disease: lessons from the clinic. Pharmacol Ther. 2007; 113(1): 121-133, doi: 10.1016/j.pharmthera.2006.07.003, indexed in Pubmed: 17000005.

2. Redhu NS, Gounni AS, Redhu NS, et al. Proinflammatory and Th2 cytokines regulate the high affinity IgE receptor (FcepsilonRI) and IgE-dependant activation of human airway smooth muscle cells. PLoS One. 2009; 4(7): e6153-94, doi: 10.1371/journal.pone.0006153, indexed in Pubmed: 19582151.

3. Roth M, Zhong J, Zumkeller C, et al. The role of IgE-receptors in IgE-dependent airway smooth muscle cell remodelling. PLoS One. 2013; 8(2): e56015, doi: 10.1371/journal. pone.0056015, indexed in Pubmed: 23457493.

4. Brusselle G, Michils A, Louis R, et al. „Real-life” effectiveness of omalizumab in patients with severe persistent allergic asthma: The PERSIST study. Respir Med. 2009; 103(11): 16331642, doi: 10.1016/j.rmed.2009.06.014, indexed in Pubmed: 19619998.

5. Humbert M, Beasley R, Ayres J, et al. Benefits of omalizumab as add-on therapy in patients with severe persistent asthma who are inadequately controlled despite best available therapy (GINA 2002 step 4 treatment): INNOVATE. Allergy. 2005; 60(3): 309-316, doi: 10.1111/j.1398-9995.2004.00772.x, indexed in Pubmed: 15679715.

6. Riccio AM, Dal Negro RW, Micheletto C, et al. Omalizumab modulates bronchial reticular basement membrane thickness and eosinophil infiltration in severe persistent allergic asthma patients. Int J Immunopathol Pharmacol. 2012; 25(2): 475-484, doi: 10.1177/039463201202500217, indexed in Pubmed: 22697079.

7. Kang JiY, Kim JW, Kim JuS, et al. Inhibitory effects of anti-immunoglobulin E antibodies on airway remodeling in a murine 
model of chronic asthma. J Asthma. 2010; 47(4): 374-380, doi: 10.3109/02770901003801972, indexed in Pubmed: 20528589.

8. Niimi A, Matsumoto H, Amitani R, et al. Airway wall thickness in asthma assessed by computed tomography. Relation to clinical indices. Am J Respir Crit Care Med. 2000; 162(4 Pt 1): 1518-1523, doi: 10.1164/ajrccm.162.4.9909044, indexed in Pubmed: 11029371.

9. Kupryś-Lipińska I, Majak P, Molinska J, et al. Effectiveness of the Polish program for the treatment of severe allergic asthma with omalizumab: a single-center experience. BMC Pulm Med. 2016; 16(1): 61, doi: 10.1186/s12890-016-0224-2, indexed in Pubmed: 27117315.

10. Yamashiro T, Matsuoka S, Estépar RS, et al. Quantitative assessment of bronchial wall attenuation with thin-section CT: An indicator of airflow limitation in chronic obstructive pulmonary disease. AJR Am J Roentgenol. 2010; 195(2): 363-369, doi: 10.2214/AJR.09.3653, indexed in Pubmed: 20651191.

11. Mansur AH, Srivastava S, Mitchell V, et al. Longterm clinical outcomes of omalizumab therapy in severe allergic asthma: Study of efficacy and safety. Respir Med. 2017; 124: 36-43, doi: 10.1016/j.rmed.2017.01.008, indexed in Pubmed: 28284319.

12. Hoshino M, Ohtawa J. Effects of adding omalizumab, an anti-immunoglobulin E antibody, on airway wall thickening in asthma. Respiration. 2012; 83(6): 520-528, doi 10.1159/000334701, indexed in Pubmed: 22236804.

13. Tajiri T, Niimi A, Matsumoto H, et al. Comprehensive efficacy of omalizumab for severe refractory asthma: a time-series observational study. Ann Allergy Asthma Immunol. 2014; 113(4): 470-5.e2, doi: 10.1016/j.anai.2014.06.004, indexed in Pubmed: 24994694.

14. de Jong PA, Müller NL, Paré PD, et al. Computed tomographic imaging of the airways: relationship to structure and function. Eur Respir J. 2005; 26(1): 140-152, doi: 10.1183/09031936.05.00007105, indexed in Pubmed: 15994401.

15. Yilmaz S, Ekici A, Ekici M, et al. High-resolution computed tomography findings in elderly patients with asthma. Eur J Radiol. 2006; 59(2): 238-243, doi: 10.1016/j.ejrad.2006.03.004, indexed in Pubmed: 16621393.

16. Boulet LP, Turcotte H, Laviolette M, et al. Airway Hyperresponsiveness, Inflammation, and Subepithelial Collagen Deposition in Recently Diagnosed versus Long-standing Mild Asthma. American Journal of Respiratory and Critical Care Medicine. 2000; 162(4): 1308-1313, doi: 10.1164/ ajrccm.162.4.9910051.

17. Hoshino M, Matsuoka S, Handa H, et al. Correlation between airflow limitation and airway dimensions assessed by multidetector CT in asthma. Respir Med. 2010; 104(6): 794-800, doi: 10.1016/j.rmed.2009.12.005, indexed in Pubmed: 20053544.

18. Kasahara K, Shiba K, Ozawa T, et al. Correlation between the bronchial subepithelial layer and whole airway wall thickness in patients with asthma. Thorax. 2002; 57(3): 242-246, indexed in Pubmed: 11867829.
19. Gono H, Fujimoto K, Kawakami S, et al. Evaluation of airway wall thickness and air trapping by HRCT in asymptomatic asthma. Eur Respir J. 2003; 22(6): 965-971, indexed in Pubmed: 14680087.

20. Niimi A, Matsumoto H, Amitani R, et al. Effect of short-term treatment with inhaled corticosteroid on airway wall thickening in asthma. Am J Med. 2004; 116(11): 725-731, doi: 10.1016/j.amjmed.2003.11.026, indexed in Pubmed: 15144908.

21. Hoshino M, Ohtawa J. Effects of budesonide/formoterol combination therapy versus budesonide alone on airway dimensions in asthma. Respirology. 2012; 17(4): 639-646, doi: 10.1111/j. 1440-1843.2012.02130.x, indexed in Pubmed: 22248352.

22. Aysola RS, Hoffman EA, Gierada D, et al. Airway remodeling measured by multidetector CT is increased in severe asthma and correlates with pathology. Chest. 2008; 134(6): 1183-1191, doi: 10.1378/chest.07-2779, indexed in Pubmed: 18641116.

23. King G, Muller N, Pare P. Evaluation of Airways in Obstructive Pulmonary Disease Using High-Resolution Computed Tomography. American Journal of Respiratory and Critical Care Medicine. 1999; 159(3): 992-1004, doi: 10.1164/ajrccm.159.3.9805064.

24. Little SA. High resolution computed tomographic assessment of airway wall thickness in chronic asthma: reproducibility and relationship with lung function and severity. Thorax. 2002; 57(3): 247-253, doi: 10.1136/thorax.57.3.247.

25. Lantero S, Alessandri G, Spallarossa D, et al. Stimulation of eosinophil IgE low-affinity receptor leads to increased adhesion molecule expression and cell migration. Eur Respir J. 2000; 16(5): 940-946, indexed in Pubmed: 11153596.

26. Rajakulasingam K, Durham SR, O'Brien F, et al. Enhanced expression of high-affinity IgE receptor (Fc epsilon RI) alpha chain in human allergen-induced rhinitis with co-localization to mast cells, macrophages, eosinophils, and dendritic cells. J Allergy Clin Immunol. 1997; 100(1): 78-86, indexed in Pubmed: 9257791.

27. Busse W, Spector S, Rosén K, et al. High eosinophil count: a potential biomarker for assessing successful omalizumab treatment effects. J Allergy Clin Immunol. 2013; 132(2): 485-6.e11, doi: 10.1016/j.jaci.2013.02.032, indexed in Pubmed: 23591271.

28. Djukanović R, Wilson SJ, Kraft M, et al. Effects of treatment with anti-immunoglobulin $\mathrm{E}$ antibody omalizumab on airway inflammation in allergic asthma. Am J Respir Crit Care Med. 2004; 170(6): 583-593, doi: 10.1164/rccm.200312-1651OC, indexed in Pubmed: 15172898.

29. Humbert M, Taillé C, Mala L, et al. STELLAIR investigators. Omalizumab effectiveness in patients with severe allergic asthma according to blood eosinophil count: the STELLAIR study. Eur Respir J. 2018; 51(5), doi: 10.1183/13993003.02523-2017, indexed in Pubmed: 29545284.

30. Chipps BE, Zeiger RS, Luskin AT, et al. Baseline asthma burden, comorbidities, and biomarkers in omalizumab-treated patients in PROSPERO. Ann Allergy Asthma Immunol. 2017; 119(6): 524-532.e2, doi: 10.1016/j.anai.2017.09.056, indexed in Pubmed: 29054589. 